\title{
Synthesis and Antimicrobial Activities Evaluation of New Spiro[4.5]dec-2-enes Containing 1,2,4-Triazole, Piperidine and Sulfonamide Moieties
}

\author{
Hany Mahmoud Dalloul ${ }^{1, ~ *}$, Khaled A. El-nwairy ${ }^{2}$, Ali Zayed Shorafa ${ }^{2}$, \\ Ahmed Sulyman Abu Samaha ${ }^{3}$ \\ ${ }^{1}$ Department of Chemistry, Faculty of Applied Science, Alaqsa University of Gaza, Gaza Strip, Palestine \\ ${ }^{2}$ Ministry of Education \& Higher Education, South Remal, Gaza, Palestine \\ ${ }^{3}$ Department of Biology, Faculty of Applied Science, Alaqsa University, Gaza, Palestine \\ Email address: \\ hmdalloul60@yahoo.com (H. M. Dalloul) \\ ${ }^{*}$ Corresponding author
}

\section{To cite this article:}

Hany Mahmoud Dalloul, Khaled A. El-nwairy, Ali Zayed Shorafa, Ahmed Sulyman Abu Samaha. Synthesis and Antimicrobial Activities Evaluation of New Spiro[4.5]dec-2-enes Containing 1,2,4-Triazole, Piperidine and Sulfonamide Moieties. American Journal of Heterocyclic Chemistry. Vol. 3, No. 1, 2017, pp. 1-7. doi: 10.11648/j.ajhc.20170301.11

Received: October 28, 2016; Accepted: February 10, 2017; Published: March 1, 2017

\begin{abstract}
A new series of 1,2,4,8-tetraazaspiro[4.5]dec-2-enes containing 1,2,4-triazole, piperidine and sulfonamide moieties have been synthesized from the reaction of 4-piperidone benzoylhydrazones having 1-methyl, 1-benzyl, and 1isopropyl groups with appropriate nitrilimines. The microanalysis and spectral data (IR, ${ }^{1} \mathrm{H} N M R,{ }^{13} \mathrm{C} N M R$ and $\mathrm{MS}$ ) of the synthesized compounds are in full agreement with their molecular structure. The microbial features of the synthesized compounds were studied by a known method. Some of titled compounds exhibited significant antimicrobial activity on several strains of microbes.
\end{abstract}

Keywords: Nitrilimines, Piperidine, Sulfonamide, 1,2,4,8-tetraazaspiro[4.5]dec-2-ene

\section{Introduction}

The interest in the chemistry of hydrazonoyl halides is a consequence of the fact that they undergo a wide variety of reactions which provide routes to many of heterocyclic and spiro heterocyclic compounds [1-4]. Spiro heterocyclic compounds possess various pharmacological properties and hence their synthesis is of interest to organic chemists. Such compounds display pronounced antimicrobial [5], analgesic [6], anti-inflammatory [6], antimycobacterial [7], antifungal [8], antitumor $[9,10]$ and antiviral $[9,10]$ activities. Among these heterocycles, spiro azoles have been identified as privileged structures in medicinal chemistry and have attracted increasing interest in the recent years [11-14].

A survey of the literature has shown that compounds having azole derivatives to possess diverse biological activity and are widely used in a medicinal chemistry [15-18]. The 1,2,4-triazole derivatives has received a considerable attention in view of their diverse pharmacological activities
[19-22] such as antimicrobial [23, 24] sedative, anticonvulsant [25], anti-inflammatory properties [26] and anticancer agents. They are also use as intermediates in obtaining colour photosensitive materials as well as toners, inks and other photographic materials such as magenta coupler in a photosensitive emulsion layer [27-30]. The most developed procedure for construction of spirocompounds depends mainly on 1,3-dipolar cycloadditions to exocyclic double bonds [31]. Recently, we described a versatile and efficient one-pot synthesis of hexa and octaazadispiroheterocyclic compounds utilizing 1,4cyclohexanedione oxime or methyl hydrazones and nitrilimines, generated in situ from the corresponding hydrazonoyl halides by the action of a suitable base $[32,33]$.

In continuation of our research studies dealing with the utility of hydrazonoyl halides for the synthesis of various heterocyclic ring and spiroheterocyclic systems via 1,3dipolar cycloaddition methodology [34-40]. It was interest to study a facile synthesis of 1,2,4,8-tetraazaspiro[4.5]dec-2- 
enes from the reaction of 1-substituted-4-piperidone benzoylhydrazones with hydrazonoyl halides having sulfonamide moiety in attempt to investigate their antimicrobial activities.

\section{Material and Methods}

\subsection{Apparatus and Chemicals}

Melting points were determined using an electro thermal melting temperature apparatus and are uncorrected. The IR spectra were measured as $\mathrm{KBr}$ pellets using a Satellite 3000 Mid infrared spectrometer. ${ }^{1} \mathrm{H}$ NMR and ${ }^{13} \mathrm{C}$ NMR spectra were recorded on a Bruker AM $300 \mathrm{MHz}$ spectrometer at $\mathrm{r}$. $\mathrm{t}$. in $\mathrm{DMSO}_{-} \mathrm{d}_{6}$ solution $\mathrm{u}$ sing tetramethylsilane (TMS) as internal reference. Chemical shifts are expressed in $\delta$ (ppm) downfield from TMS and coupling constants are in Hertz $(\mathrm{Hz})$. Electron impact (EI) mass spectra were run on a Shimadzu GCMS-QP1000 EX spectrometer at $70 \mathrm{eV}$. Elemental analysis were carried out at micro analytical laboratory, Cairo University, Cairo, Egypt. 1-Methyl, 1benzyl and 1-isopropyl-4-piperidone was purchased from Avocado Research Chemicals, England, and used without further purification. Hydrazonoyl chlorides 1 employed in this study, were prepared via direct coupling of the appropriate sulfa drug diazonium chloride with 3chloroacetylacetone or phenacyl chloride or $\alpha$ chloroacetoacetanilide or $\alpha$-bromo-2-acetylfuran or $\alpha$-bromo2 -acetylthiophene or $\omega$-bromoacetonaphthone in ethanolic sodium acetate solution following standard procedures [41]. 1-substituted-4-piperidone benzoylhydrazones 3 were obtained by reacting the appropriate 1-substituted-4piperidone with benzolyhydrazine in excess following reported procedures [37, 42].

\subsection{Synthesis of 1,2,4,8-tetraazspiro[4.5]dec-2-enes (General Procedure)}

To a stirred solution of hydrazonoyl halides 1 (5 mmol) and 1-substituted-4-piperidone hydrazones $3(10 \mathrm{mmol})$ in 1,4-dioxane $(50-70 \mathrm{ml})$, triethylamine $(5 \mathrm{mmol})$ in 1,4dioxane $(10 \mathrm{ml})$ dropwise added at room temperature. Stirring was continued to reaction completion for 12-16 hours (monitoring the reaction progress by TLC). The solvent was then removed under vacuum, and the residual solid was washed with water $(100 \mathrm{ml})$ to get rid of the triethylamine salt. In some cases the residue was extracted with chloroform $(3 \times 30 \mathrm{ml})$ and the combined extracts were washed with water $(50 \mathrm{ml})$, dried over anhydrous sodium sulfate. The solvent $\left(\mathrm{CHCl}_{3}\right)$ was evaporated in vacuum, and the crude product was triturated with ethanol $(10-20 \mathrm{ml})$. The crude solid products were collected and recrystallized from appropriate solvents to afford the desired compounds $4 \mathrm{a}-\mathrm{u}$. The following compounds were prepared using this method:

3-Acetyl-4-benzoylamino-8-methyl-l-[4-(thiazol-2-ylsulfonyl)phenyl]-l,2,4,8-tetraazaspiro-[4.5]dec-2-ene 4a: Yield $75 \%$, m.p. $188-190^{\circ} \mathrm{C}$ (ethanol). IR, v/cm ${ }^{-1}$ : 3380, 3347, 2365 (NH's), 1689, 1675 (C=O's), $1620(\mathrm{C}=\mathrm{N}), 1347$,
$1156\left(\mathrm{SO}_{2}\right) ;{ }^{1} \mathrm{H} \mathrm{NMR}, \delta / \mathrm{ppm}: 11.61\left(\mathrm{~s}, 1 \mathrm{H}, \mathrm{SO}_{2} \mathrm{NH}\right), 9.45$ (s, $1 \mathrm{H}, \mathrm{CONH}), 8.76(\mathrm{~d}, 1 \mathrm{H}$ thiazole ring), 7.38-7.21 (m, 9H, Ar-H), 6.62 (d, $1 \mathrm{H}$ thiazole ring), 2.82-1.80 (m, 8H, 4CH $)$ $2.56\left(\mathrm{~s}, 3 \mathrm{H}, \mathrm{CH}_{3} \mathrm{CO}\right), 2.41\left(\mathrm{~s}, 3 \mathrm{H}, \mathrm{NCH}_{3}\right) ;{ }^{13} \mathrm{C} \quad \mathrm{NMR}$ $\left(\mathrm{DMSO}_{6}\right)$ : $\delta / \mathrm{ppm} 189.60(\mathrm{C}=\mathrm{O}), 169.01(\mathrm{~N}-\mathrm{C}=\mathrm{O}), 147.34$ $(\mathrm{C}=\mathrm{N}$ ), 166.7-115.20 (Ar-C and thiazole-C), 91.12 (spiro carbon), $52.89\left(2 \mathrm{CH}_{2}\right), 46.60\left(\mathrm{NCH}_{3}\right), 32.20\left(2 \mathrm{CH}_{2}\right), 26.25$ $\left(\mathrm{CH}_{3}\right)$. MS: $\left(\mathrm{M}^{+}=343\right)$; Anal. Calcd for $\mathrm{C}_{18} \mathrm{H}_{22} \mathrm{~N}_{6} \mathrm{O}_{3} \mathrm{~S}_{2}$ (434.54): C 49.75\%, H 5.10\%, N 19.34\%. Found: C 49.56\%, H $4.98 \%$, N $19.47 \%$.

3-Acetyl-4-benzoylamino-8-isopropyl-l-[4-(thiazol-2-ylsulfonyl)phenyl]-l,2,4,8-tetraazaspiro[4.5]-dec-2-ene $4 b$ : Yield $72 \%$, m.p. $193-195{ }^{\circ} \mathrm{C}$ (ethanol). IR, v/ $\mathrm{crn}^{-1}: 3377$, 3345, 2362 (NH's), 1687, $1665\left(\mathrm{C}=\mathrm{O}^{\prime} \mathrm{s}\right), 1621(\mathrm{C}=\mathrm{N}), 1346$, $1154\left(\mathrm{SO}_{2}\right) ;{ }^{1} \mathrm{H} \mathrm{NMR}, \delta / \mathrm{ppm}: 11.64\left(\mathrm{~s}, 1 \mathrm{H}, \mathrm{SO}_{2} \mathrm{NH}\right), 9.46$ (s, $1 \mathrm{H}, \mathrm{CONH}), 8.72(\mathrm{~d}, 1 \mathrm{H}$ thiazole ring), 7.36-7.20 (m, $9 \mathrm{H}$, Ar-H), 6.64 (d, $1 \mathrm{H}$ thiazole ring), 2.86-1.82 (m, $\left.8 \mathrm{H}, 4 \mathrm{CH}_{2}\right)$ $2.56\left(\mathrm{~s}, 3 \mathrm{H}, \mathrm{CH}_{3} \mathrm{CO}\right), 2.43(1 \mathrm{H}, \mathrm{m}, \mathrm{CH}), 1.25\left(6 \mathrm{H}, \mathrm{d}, 2 \mathrm{CH}_{3}\right)$. ${ }^{13} \mathrm{C}$ NMR (DMSO-d $\left.)_{6}\right): \delta /$ ppm $189.60(\mathrm{C}=\mathrm{O}), 169.05(\mathrm{~N}-$ $\mathrm{C}=\mathrm{O}), 147.34(\mathrm{C}=\mathrm{N}), 166.7-115.20($ Ar- $\mathrm{C}$ and thiazole-C), 91.05 (spiro carbon), $52.87\left(2 \mathrm{CH}_{2}\right), 47.60(\mathrm{NCH}), 32.20$ $\left(2 \mathrm{CH}_{2}\right), 27.80\left(2 \mathrm{CH}_{3}\right), 26.24\left(\mathrm{CH}_{3}\right)$. MS: $\left(\mathrm{M}^{+} \cdot=462\right)$; Anal. Calcd. for $\mathrm{C}_{20} \mathrm{H}_{26} \mathrm{~N}_{6} \mathrm{O}_{3} \mathrm{~S}_{2}$ (462.60): C 51.93\%, H 5.67\%, N $18.17 \%$. Found: C 52.18\%, H 5.80\%, N 18.05\%.

3-Acetyl-4-benzoylamino-8-benzyl-l-[4-(thiazol-2-ylsulfonyl)phenyl]-l,2,4,8-tetraazaspiro-[4.5]dec-2-ene 4c: Yield $75 \%$, m.p. $176-178^{\circ} \mathrm{C}$ (ethanol). IR, v/cm ${ }^{-1}$ : 3375, 3344, 2360 (NH's), 1689, 1665 (C=O's), $1622(\mathrm{C}=\mathrm{N}), 1347$, $1153\left(\mathrm{SO}_{2}\right) ;{ }^{1} \mathrm{H} \mathrm{NMR}, \delta / \mathrm{ppm}: 11.61\left(\mathrm{~s}, 1 \mathrm{H}, \mathrm{SO}_{2} \mathrm{NH}\right), 9.44$ (s, $1 \mathrm{H}, \mathrm{CONH}), 8.76(\mathrm{~d}, 1 \mathrm{H}$ thiazole ring), 7.38-7.21 (m, $14 \mathrm{H}$, Ar-H), 6.62 (d, $1 \mathrm{H}$ thiazole ring), $3.26\left(2 \mathrm{H}, \mathrm{s}, \mathrm{CH}_{2} \mathrm{Ph}\right), 2.82-$ $1.83\left(\mathrm{~m}, 8 \mathrm{H}, 4 \mathrm{CH}_{2}\right) 2.56\left(\mathrm{~s}, 3 \mathrm{H}, \mathrm{CH}_{3} \mathrm{CO}\right) .{ }^{13} \mathrm{C}$ NMR (DMSO$\left.\mathrm{d}_{6}\right): \delta / p p m 189.60(\mathrm{C}=\mathrm{O}), 169.02(\mathrm{~N}-\mathrm{C}=\mathrm{O}), 147.34(\mathrm{C}=\mathrm{N})$, 166.97-115.24 (Ar-C and thiazole-C), 91.10 (spiro carbon), $52.80\left(2 \mathrm{CH}_{2}\right), 50.10\left(\mathrm{CH}_{2} \mathrm{Ph}\right), 32.22\left(2 \mathrm{CH}_{2}\right), 26.16\left(\mathrm{CH}_{3}\right)$. MS: $\left(\mathrm{M}^{+}=510\right)$; Anal. Calcd for $\mathrm{C}_{24} \mathrm{H}_{26} \mathrm{~N}_{6} \mathrm{O}_{3} \mathrm{~S}_{2}(510.64)$ : $\mathrm{C}$ $56.45 \%$, H 5.13\%, N 16.46\%. Found:C 56.20\%, H 5.02\%, N $16.33 \%$.

3-Acetyl-4-benzoylamino-8-methyl-l-[4-(pyrimidin-2-ylsulfonyl)phenyl]-l,2,4,8-tetraazaspiro-[4.5]-dec-2-ene 4d: Yield $70 \%$, m.p. $182-184{ }^{\circ} \mathrm{C}$ (ethanol). IR, v/cm ${ }^{-1}: 3380$, 3346, 2360 (NH's), 1690, 1660 (C=O's), $1624(\mathrm{C}=\mathrm{N}), 1344$, $1152\left(\mathrm{SO}_{2}\right)$; ${ }^{1} \mathrm{H} \mathrm{NMR}, \delta / \mathrm{ppm}: 11.65\left(\mathrm{~s}, 1 \mathrm{H}, \mathrm{SO}_{2} \mathrm{NH}\right), 9.52(\mathrm{~s}$, $1 \mathrm{H}, \mathrm{CONH}), 8.86(\mathrm{~d}, 2 \mathrm{H}$ pyrimidine ring $), 7.37-7.18(\mathrm{~m}, 9 \mathrm{H}$, Ar-H), 6.89 (t, $1 \mathrm{H}$ pyrimidine ring), 2.81-1.80 (m, $\left.8 \mathrm{H}, 4 \mathrm{CH}_{2}\right)$ $2.55\left(\mathrm{~s}, 3 \mathrm{H}, \mathrm{CH}_{3} \mathrm{CO}\right) .2 .40\left(\mathrm{~s}, 3 \mathrm{H}, \mathrm{NCH}_{3}\right) \mathrm{ppm} .{ }^{13} \mathrm{C} \mathrm{NMR}$ $\left(\mathrm{DMSO}-\mathrm{d}_{6}\right): \delta=189.82(\mathrm{C}=\mathrm{O}), 169.10(\mathrm{~N}-\mathrm{C}=\mathrm{O}), 147.62$ $(\mathrm{C}=\mathrm{N}$ ), 167.90-110.32 (Ar-C and pyrimidine-C), 91.14 (spiro carbon), $53.10\left(2 \mathrm{CH}_{2}\right), 46.62(\mathrm{NCH} 3), 32.21\left(2 \mathrm{CH}_{2}\right), 26.24$ $\left(\mathrm{CH}_{3}\right)$. MS: $\left(\mathrm{M}^{+}=429\right)$; Anal. Calcd for $\mathrm{C}_{19} \mathrm{H}_{23} \mathrm{~N}_{7} \mathrm{O}_{3} \mathrm{~S}$ (429.50): C 53.13\%, H 5.40\%, N 22.83\%. Found:C 52.90\%, H $5.53 \%$, N $22.96 \%$.

3-Acetyl-4-benzoylamino-8-isopropyl-l-[4-(pyrimidin-2-ylsulfonyl)phenyl]-l,2,4,8-tetraazaspiro-[4.5]dec-2-ene 4e: Yield $77 \%$, m.p. $202-204{ }^{\circ} \mathrm{C}$ (methanol). IR, v/cm ${ }^{-1}: 3370$, 3342, 2364 (NH's), 1688, $1662(\mathrm{C}=\mathrm{O}$ 's), $1621(\mathrm{C}=\mathrm{N}), 1340$, $1151\left(\mathrm{SO}_{2}\right) ;{ }^{1} \mathrm{H} \mathrm{NMR}, \delta / \mathrm{ppm}: 11.65$ (s, $\left.1 \mathrm{H}, \mathrm{SO}_{2} \mathrm{NH}\right), 9.52$ (s, 
$1 \mathrm{H}, \mathrm{CONH}), 8.86(\mathrm{~d}, 2 \mathrm{H}$ pyrimidine ring), 7.37-7.18 (m, 9H, Ar-H), 6.89 (t, $1 \mathrm{H}$ pyrimidine ring), 2.86-1.83 (m, $\left.8 \mathrm{H}, 4 \mathrm{CH}_{2}\right)$ $2.56\left(\mathrm{~s}, 3 \mathrm{H}, \mathrm{CH}_{3} \mathrm{CO}\right) 2.35(\mathrm{~m}, 1 \mathrm{H}, \mathrm{CH}), 1.26\left(\mathrm{~s}, 6 \mathrm{H}, 2 \mathrm{CH}_{3}\right)$; ${ }^{13} \mathrm{C}$ NMR $\left(\mathrm{DMSO}-\mathrm{d}_{6}\right): \delta=189.76(\mathrm{C}=\mathrm{O}), 169.05(\mathrm{~N}-\mathrm{C}=\mathrm{O})$, $147.66(\mathrm{C}=\mathrm{N}), 167.87-110.23$ (Ar-C and pyrimidine-C), 91.10 (spiro carbon), $53.12\left(2 \mathrm{CH}_{2}\right), 47.60(\mathrm{CH}), 32.20$ $\left(2 \mathrm{CH}_{2}\right), 27.45\left(2 \mathrm{CH}_{3}\right)$. MS: $\left(\mathrm{M}^{+\cdot}=457\right)$; Anal. Calcd for $\mathrm{C}_{21} \mathrm{H}_{27} \mathrm{~N}_{7} \mathrm{O}_{3} \mathrm{~S}$ (457.56): C 55.13\%, H 5.95\%, N 21.43\%. Found: C 54.90\%, H 6.05, N 21.30\%.

3-Acetyl-4-benzoylamino-8-benzyl-l-[4-(pyrimidin-2-ylsulfonyl)phenyl]-l,2,4,8-tetraazaspiro[4.5]-dec-2-ene $4 f$ : Yield $75 \%$, m.p. $216-218{ }^{\circ} \mathrm{C}$ (methanol). IR, v/cm ${ }^{-1}: 3378$, 3344, 2358 (NH's), 1689, $1662(\mathrm{C}=\mathrm{O} ' \mathrm{~s}), 1620(\mathrm{C}=\mathrm{N}), 1345$, $1150\left(\mathrm{SO}_{2}\right) ;{ }^{1} \mathrm{H} \mathrm{NMR}, \delta / \mathrm{ppm}$ : $11.65\left(\mathrm{~s}, 1 \mathrm{H}, \mathrm{SO}_{2} \mathrm{NH}\right), 9.48(\mathrm{~s}$, $1 \mathrm{H}, \mathrm{CONH}), 8.86$ (d, 2H pyrimidine ring), 7.37-7.18 (m, 14H, Ar-H), 6.89 (t, 1H pyrimidine ring), $3.25(\mathrm{~s}, 2 \mathrm{H}$, $\left.\mathrm{PhCH}_{2}\right), 2.86-1.83\left(\mathrm{~m}, 8 \mathrm{H}, 4 \mathrm{CH}_{2}\right) 2.56\left(\mathrm{~s}, 3 \mathrm{H}, \mathrm{CH}_{3} \mathrm{CO}\right) .{ }^{13} \mathrm{C}$ NMR $\left(\mathrm{DMSO}-\mathrm{d}_{6}\right): \delta=189.62(\mathrm{C}=\mathrm{O}), 169.10(\mathrm{~N}-\mathrm{C}=\mathrm{O})$, $147.64(\mathrm{C}=\mathrm{N}), 167.90-110.32$ (Ar-C and pyrimidine-C), 91.12 (spiro carbon), $53.16\left(2 \mathrm{CH}_{2}\right), 50.05\left(\mathrm{PhCH}_{2}\right), 32.24$ $\left(2 \mathrm{CH}_{2}\right), 26.21\left(\mathrm{CH}_{3}\right)$. MS: $\left(\mathrm{M}^{+}=505\right)$; Anal. Calcd. for $\mathrm{C}_{25} \mathrm{H}_{27} \mathrm{~N}_{7} \mathrm{O}_{3} \mathrm{~S}$ (505.60): C 59.39\%, H 5.38\%, N 19.39\%. Found: C 59.60\%, H 5.26\%, N 19.27\%.

3-Benzoyl-4-benzoylamino-8-methyl-l-[4-(thiazol-2-ylsulfonyl)phenyl]-l,2,4,8-tetraazaspiro[4.5]-dec-2-ene 4g: Yield $72 \%$, m.p. $234-236{ }^{\circ} \mathrm{C}$ (methanol). IR, v/cm ${ }^{-1}$ : 3367, 3342, 2357 (NH's), 1655, 1660 (C=O's), $1615(\mathrm{C}=\mathrm{N}), 1346$, $1152\left(\mathrm{SO}_{2}\right) ;{ }^{1} \mathrm{H} \mathrm{NMR}, \delta / \mathrm{ppm}$ : $11.61\left(\mathrm{~s}, 1 \mathrm{H}, \mathrm{SO}_{2} \mathrm{NH}\right), 9.43$ (s, $1 \mathrm{H}, \mathrm{CONH}), 8.76(\mathrm{~d}, 1 \mathrm{H}$ thiazole ring), 7.38-7.21 (m, $14 \mathrm{H}$, Ar-H), 6.62 (d, $1 \mathrm{H}$ thiazole ring), 2.82-1.83 (m, $\left.8 \mathrm{H}, 4 \mathrm{CH}_{2}\right)$ $2.36\left(\mathrm{~s}, 3 \mathrm{H}, \mathrm{NCH}_{3}\right) .{ }^{13} \mathrm{C} \mathrm{NMR}\left(\mathrm{DMSO}-\mathrm{d}_{6}\right): \delta / \mathrm{ppm} 184.64$ $(\mathrm{Ar}-\mathrm{C}=\mathrm{O}), 168.85(\mathrm{~N}-\mathrm{C}=\mathrm{O}), 147.78(\mathrm{C}=\mathrm{N}), 167.27-115.21$ (Ar-C and thiazole-C), 90.85 (spiro carbon), $53.102\left(2 \mathrm{CH}_{2}\right)$, $46.65\left(\mathrm{NCH}_{3}\right), 32.10\left(2 \mathrm{CH}_{2}\right)$. MS: $\left(\mathrm{M}^{+}=496\right)$; Anal. Calcd for $\mathrm{C}_{23} \mathrm{H}_{24} \mathrm{~N}_{6} \mathrm{O}_{3} \mathrm{~S}_{2}$ (496.61): C 55.63\%, H 4.87\%, N 16.92\%. Found: C 55.40\%, H $4.76 \%$, N $17.06 \%$.

3-Benzoyl-4-benzoylamino-8-isopropyl-l-[4-(thiazol-2-ylsulfonyl)phenyl]-l,2,4,8-tetraazaspiro[4.5]-dec-2-ene 4h: Yield $68 \%$, m.p. $225-227{ }^{\circ} \mathrm{C}$ (ethanol). IR, v/cm ${ }^{-1}$ : 3365, 3341, 2355 (NH's), 1656, $1661(\mathrm{C}=\mathrm{O}$ 's), $1612(\mathrm{C}=\mathrm{N}), 1345$, $1152\left(\mathrm{SO}_{2}\right) ;{ }^{1} \mathrm{H} \mathrm{NMR}, \delta / \mathrm{ppm}: 11.61\left(\mathrm{~s}, 1 \mathrm{H}, \mathrm{SO}_{2} \mathrm{NH}\right), 9.41(\mathrm{~s}$, $1 \mathrm{H}, \mathrm{CONH}), 8.76(\mathrm{~d}, 1 \mathrm{H}$ thiazole ring), 7.38-7.21 (m, $14 \mathrm{H}$, Ar-H), 6.62 (d, $1 \mathrm{H}$ thiazole ring), 2.90-1.68 (m, 8H, 4CH $)$ $2.42(\mathrm{~s}, 3 \mathrm{H}, \mathrm{CH}), 1.3\left(\mathrm{~s}, 6 \mathrm{H}, 3 \mathrm{CH}_{3}\right) ;{ }^{13} \mathrm{C} \mathrm{NMR}\left(\mathrm{DMSO}-\mathrm{d}_{6}\right)$ : $\delta /$ ppm $184.60(\mathrm{Ar}-\mathrm{C}=\mathrm{O}), 168.56(\mathrm{~N}-\mathrm{C}=\mathrm{O}), 147.84(\mathrm{C}=\mathrm{N})$, 167.17-115.14 (Ar-C and thiazole-C), 90.90 (spiro carbon), $53.20\left(2 \mathrm{CH}_{2}\right), 47.60(\mathrm{CH}), 31.80\left(2 \mathrm{CH}_{2}\right), 27.46\left(2 \mathrm{CH}_{3}\right) . \mathrm{MS}$ : $\left(\mathrm{M}^{+}=524\right)$; Anal. Calcd for $\mathrm{C}_{25} \mathrm{H}_{28} \mathrm{~N}_{6} \mathrm{O}_{3} \mathrm{~S}_{2} \quad(524.67)$ : $\mathrm{C}$ $57.23 \%$, H 5.38\%, N 16.02\%. Found: C 57.50\%, H 5.50\%, N $15.90 \%$.

3-Benzoyl-4-benzoylamino-8-benzyl-l-[4-(thiazol-2-ylsulfonyl)phenyl]-l,2,4,8-tetraazaspiro[4.5]-dec-2-ene 4i: Yield $66 \%$, m.p. $213-215{ }^{\circ} \mathrm{C}$ (ethanol). IR, v/crn ${ }^{-1}$ : 3365, 3338, 2356 (NH's), 1654, 1665 (C=O's), $1614(\mathrm{C}=\mathrm{N}), 1344$, $1153\left(\mathrm{SO}_{2}\right) ;{ }^{1} \mathrm{H} \mathrm{NMR}, \delta / \mathrm{ppm}$ : $11.61\left(\mathrm{~s}, 1 \mathrm{H}, \mathrm{SO}_{2} \mathrm{NH}\right), 9.40$ (s, $1 \mathrm{H}, \mathrm{CONH}), 8.76(\mathrm{~d}, 1 \mathrm{H}$ thiazole ring), 7.38-7.21 (m, $19 \mathrm{H}$, $\mathrm{Ar}-\mathrm{H}), 6.62$ (d, $1 \mathrm{H}$ thiazole ring), 3.36 (s, 2H, $\mathrm{PhCH}_{3}$ ), 2.82- $1.83\left(\mathrm{~m}, 8 \mathrm{H}, 4 \mathrm{CH}_{2}\right) \cdot{ }^{13} \mathrm{C}$ NMR (DMSO-d 6 ): $\delta / \mathrm{ppm} 184.52$ $(\mathrm{Ar}-\mathrm{C}=\mathrm{O}), 168.72(\mathrm{~N}-\mathrm{C}=\mathrm{O}), 147.81(\mathrm{C}=\mathrm{N}), 167.12-115.18$ (Ar-C and thiazole-C), 90.82 (spiro carbon), $53.10\left(2 \mathrm{CH}_{2}\right)$, $50.05\left(\mathrm{PhCH}_{2}\right), 31.86\left(2 \mathrm{CH}_{2}\right)$. MS: $\left(\mathrm{M}^{+}=572\right)$; Anal. Calcd for $\mathrm{C}_{29} \mathrm{H}_{28} \mathrm{~N}_{6} \mathrm{O}_{3} \mathrm{~S}_{2}$ (572.71): C 60.82\%, H 4.93\%, N $14.67 \%$. Found: C $61.06 \%$, H 5.05\%, N 14.55\%.

4-Benzoylamino-8-methyl-3-phenylaminocarbonyl-l-[4(thiazol-2-yl-sulfonyl)phenyl]-l,2,4,8-tetra-azaspiro[4.5]dec2-ene $4 j$ : Yield $72 \%$, m.p. $238-240^{\circ} \mathrm{C}$ (ethanol). IR, $\mathrm{v} / \mathrm{cm}^{-1}$ : 3368, 3340, 2354 (NH's), 1665, 1670 (C=O's), $1615(\mathrm{C}=\mathrm{N})$, 1345, $1144\left(\mathrm{SO}_{2}\right) ;{ }^{1} \mathrm{H} \mathrm{NMR}, \delta / \mathrm{ppm}: 11.72\left(\mathrm{~s}, 1 \mathrm{H}, \mathrm{SO}_{2} \mathrm{NH}\right)$, $9.86(1 \mathrm{H}, \mathrm{s}, \mathrm{Ph}-\mathrm{NH}), 9.47(\mathrm{~s}, 1 \mathrm{H}, \mathrm{CONH}), 8.76(\mathrm{~d}, 1 \mathrm{H}$ thiazole ring), 7.38-7.21 (m, 14H, Ar-H), $6.62(\mathrm{~d}, 1 \mathrm{H}$ thiazole ring), 2.92-1.65 (m, 8H, 4CH $) 2.40\left(\mathrm{~s}, 3 \mathrm{H}, \mathrm{NCH}_{3}\right) .{ }^{13} \mathrm{C} \mathrm{NMR}$ $\left(\mathrm{DMSO}-\mathrm{d}_{6}\right)$ : $\delta / \mathrm{ppm}: 168.62(\mathrm{~N}-\mathrm{C}=\mathrm{O}), 159.80($ amide $\mathrm{C}=\mathrm{O})$, $147.37(\mathrm{C}=\mathrm{N}), 166.70-115.22($ Ar- $\mathrm{C}$ and thiazole-C), 90.12 (spiro carbon), $52.89\left(2 \mathrm{CH}_{2}\right), 46.60\left(\mathrm{NCH}_{3}\right), 31.94\left(2 \mathrm{CH}_{2}\right)$. MS: $\left(\mathrm{M}^{+} \cdot=511\right)$; Anal. Calcd for $\mathrm{C}_{23} \mathrm{H}_{25} \mathrm{~N}_{7} \mathrm{O}_{3} \mathrm{~S}_{2}$ (511.63): $\mathrm{C}$ $54.00 \%$, H $4.93 \%$, N 19.19\%. Found: C 53.77\%, H 5.05\%, N $19.30 \%$.

4-Benzoylamino-8-benzyl-3-phenylaminocarbonyl-l-[4(thiazol-2-yl-sulfonyl)phenyl]-l,2,4,8-tetra-azaspiro[4.5]dec2-ene 4k: Yield 70\%, m.p. 229-231 ${ }^{\circ} \mathrm{C}$ (ethanol). IR, v/crn ${ }^{-1}$ : 3366, 3347, 2356 (NH's), 1660, 1668 (C=O's), $1618(\mathrm{C}=\mathrm{N})$, 1342, $1150\left(\mathrm{SO}_{2}\right) ;{ }^{1} \mathrm{H} \mathrm{NMR}, \delta / \mathrm{ppm}: 11.61\left(\mathrm{~s}, 1 \mathrm{H}, \mathrm{SO}_{2} \mathrm{NH}\right)$, $9.88(1 \mathrm{H}, \mathrm{s}, \mathrm{Ph}-\mathrm{NH}), 9.48(\mathrm{~s}, 1 \mathrm{H}, \mathrm{CONH}), 8.76(\mathrm{~d}, 1 \mathrm{H}$ thiazole ring), 7.38-7.21 (m, 19H, Ar-H), $6.62(\mathrm{~d}, 1 \mathrm{H}$ thiazole ring), $3.40\left(\mathrm{~s}, 2 \mathrm{H} \mathrm{PhCH}_{2}\right), 2.86-1.84\left(\mathrm{~m}, 8 \mathrm{H}, 4 \mathrm{CH}_{2}\right) ;{ }^{13} \mathrm{C}$ NMR (DMSO-d $)_{6}$ ): $\delta / p p m: 168.53(\mathrm{~N}-\mathrm{C}=\mathrm{O}), 159.56$ (amide $\mathrm{C}=\mathrm{O}), 147.34(\mathrm{C}=\mathrm{N}), 166.67-115.23$ (Ar-C and thiazole-C), 89.95 (spiro carbon), $52.87\left(2 \mathrm{CH}_{2}\right), 49.78\left(\mathrm{PhCH}_{2}\right), 31.90$ $\left(2 \mathrm{CH}_{2}\right)$. MS: $\left(\mathrm{M}^{+}=587\right)$; Anal. Calcd for $\mathrm{C}_{29} \mathrm{H}_{29} \mathrm{~N}_{7} \mathrm{O}_{3} \mathrm{~S}_{2}$ (587.73): C 74.97\%, H 6.29\%, N 14.57\%. Found: C 75.22\%, H $6.17 \%$, N $14.68 \%$.

4-Benzoylamino-8-Isopropyl-3-phenylaminocarbonyl-l-[4(pyrimidin-2-yl-sulfonyl)phenyl]-l,2,4,8-tetraazaspiro[4.5]dec-2-ene 41: Yield $75 \%$, m.p. $234-236^{\circ} \mathrm{C}$ (methanol). IR, $\mathrm{v} / \mathrm{crn}^{-1}$ : 3370, 3345, 2357 (NH's), 1665, $1672(\mathrm{C}=\mathrm{O}), 1619$ $(\mathrm{C}=\mathrm{N}), 1348,1153\left(\mathrm{SO}_{2}\right) ;{ }^{1} \mathrm{H} \mathrm{NMR}, \delta / \mathrm{ppm}: 11.60(\mathrm{~s}, 1 \mathrm{H}$, $\mathrm{SO}_{2} \mathrm{NH}$ ), 9.84 (s, 1H, PhNH), 9.45 (s, 1H, CONH), 8.86 (d, $2 \mathrm{H}$ pyrimidine ring), 7.37-7.18 (m, 14H, Ar-H), $6.89(\mathrm{t}, 1 \mathrm{H}$ pyrimidine ring), $2.41(\mathrm{~s}, \mathrm{H}, \mathrm{CH}), 1.29\left(\mathrm{~s}, 6 \mathrm{H}, 2 \mathrm{CH}_{3}\right) ;{ }^{13} \mathrm{C}$ NMR (DMSO-d $\left.{ }_{6}\right): \delta / p p m: 168.80(\mathrm{~N}-\mathrm{C}=\mathrm{O}), 159.65$ (amide $\mathrm{C}=\mathrm{O}), 143.6(\mathrm{C}=\mathrm{N}), 167.9-110.3($ Ar- $\mathrm{C}$ and pyrimidine-C), 89.97 (spiro carbon), $52.90\left(2 \mathrm{CH}_{2}\right), 47.50(\mathrm{CH}), 31.80$ $\left(2 \mathrm{CH}_{2}\right), 27.44\left(2 \mathrm{CH}_{3}\right)$. MS: $\left(\mathrm{M}^{+}=534\right)$; Anal. Calcd for $\mathrm{C}_{26} \mathrm{H}_{30} \mathrm{~N}_{8} \mathrm{O}_{3} \mathrm{~S}$ (534.64): C 58.41\%, H 5.66\%, N 20.96\%. Found: C 58.65\%, H 5.75\%, N 21.10\%.

4-Benzoylamino-8-benzyl-3-phenylaminocarbonyl-l-[4(pyrimidin-2-yl-sulfonyl)phenyl]-l,2,4,8-tetraazaspiro[4.5]dec-2-ene 4m: Yield 74\%, m.p. 242-244 ${ }^{\circ} \mathrm{C}$ (ethanol). IR, $\mathrm{v} / \mathrm{cm}^{-1}$ : 3372, 3347, 2359 (NH's), 1665, $1670(\mathrm{C}=\mathrm{O}), 1613$ $(\mathrm{C}=\mathrm{N}), 1345,1155\left(\mathrm{SO}_{2}\right) ;{ }^{1} \mathrm{H} \mathrm{NMR}, \delta / \mathrm{ppm}: 11.60(\mathrm{~s}, 1 \mathrm{H}$, $\left.\mathrm{SO}_{2} \mathrm{NH}\right), 9.84$ (s, 1H, PhNH), 9.46 (s, 1H, CONH), 8.86 (d, $2 \mathrm{H}$ pyrimidine ring), 7.37-7.18 (m, 14H, Ar-H), $6.89(\mathrm{t}, 1 \mathrm{H}$ pyrimidine ring), $3.41\left(\mathrm{~s}, 2 \mathrm{H}, \mathrm{PhCH}_{2}\right) ;{ }^{13} \mathrm{C}$ NMR (DMSO$\mathrm{d}_{6}$ ): $\delta /$ ppm: $168.65(\mathrm{~N}-\mathrm{C}=\mathrm{O}), 159.68$ (amide $\mathrm{C}=\mathrm{O}$ ), 146.89 
$(\mathrm{C}=\mathrm{N}), 167.92-110.34$ (Ar-C and pyrimidine-C), 89.96 (spiro carbon), $52.96\left(2 \mathrm{CH}_{2}\right), 49.60\left(\mathrm{PhCH}_{2}\right), 31.78\left(2 \mathrm{CH}_{2}\right)$. MS: $\left(\mathrm{M}^{+}=\right.$582); Anal. Calcd for $\mathrm{C}_{30} \mathrm{H}_{30} \mathrm{~N}_{8} \mathrm{O}_{3} \mathrm{~S}$ (582.69): $\mathrm{C}$ $61.84 \%$, H 5.19\%, N 19.23\%. Found: C 62.10\%, H 5.06\%, N $19.34 \%$.

4-Benzoylamino-3-(2-furyl)-8-methyl-l-[4-(thiazol-2-ylsulfonyl)phenyl]-l,2,4,8-tetraazaspiro[4.5]-dec-2-ene 4n: Yield 63\%), m.p. $193-195{ }^{\circ} \mathrm{C}$ (methanol). IR, v/cm ${ }^{-1}: 3375$, 3337, 2361 (NH's), 1660, 1667 (C=O's), $1620(\mathrm{C}=\mathrm{N}), 1351$, $1154\left(\mathrm{SO}_{2}\right) ;{ }^{1} \mathrm{H} \mathrm{NMR}, \delta / \mathrm{ppm}: 11.61\left(\mathrm{~s}, 1 \mathrm{H}, \mathrm{SO}_{2} \mathrm{NH}\right), 9.42(\mathrm{~s}$, $1 \mathrm{H}, \mathrm{CONH}), 8.76(\mathrm{~d}, 1 \mathrm{H}$ thiazole ring $), 8.23-7.22(\mathrm{~m}, 12 \mathrm{H}$, $\mathrm{Ar}-\mathrm{H}), 6.62\left(\mathrm{~d}, 1 \mathrm{H}\right.$ thiazole ring), 2.84-1.81 (m, $\left.8 \mathrm{H}, 4 \mathrm{CH}_{2}\right)$ $2.34\left(\mathrm{~s}, 3 \mathrm{H}, \mathrm{NCH}_{3}\right) ;{ }^{13} \mathrm{C} \mathrm{NMR}\left(\mathrm{DMSO}-\mathrm{d}_{6}\right): \delta / \mathrm{ppm} 173.68$ $(\mathrm{Ar}-\mathrm{C}=\mathrm{O}), \quad 169.24 \quad(\mathrm{~N}-\mathrm{C}=\mathrm{O}), \quad 147.30 \quad(\mathrm{C}=\mathrm{N}), \quad 166.65-$ 115.16(Ar-C and thiazole-C), 90.42 (spiro carbon), 52.90 $\left(2 \mathrm{CH}_{2}\right), 46.68\left(\mathrm{NCH}_{3}\right), 31.78\left(2 \mathrm{CH}_{2}\right)$. MS: $\left(\mathrm{M}^{+} \cdot=486\right)$; Anal. Calcd for $\mathrm{C}_{21} \mathrm{H}_{22} \mathrm{~N}_{6} \mathrm{O}_{4} \mathrm{~S}_{2}$ (486.58): C 51.84\%, H 4.56\%, N $17.27 \%$. Found: C $52.11 \%$, H $4.70 \%$, N $17.41 \%$.

4-Benzoylamino-3-(2-furyl)-8-isopropyl-l-[4-(thiazol-2-ylsulfonyl)phenyl]-l,2,4,8-tetraazaspiro[4.5]-dec-2-ene 4o: Yield $65 \%$, m.p. $210-212^{\circ} \mathrm{C}$ (methanol). IR, v / c m ${ }^{-1}: 3370$, 3335, 2360 (NH's), 1662, 1669 (C=O's), $1618(\mathrm{C}=\mathrm{N}), 1349$, $1151\left(\mathrm{SO}_{2}\right) ;{ }^{1} \mathrm{H}$ NMR, $\delta / \mathrm{PPM}: 11.61\left(\mathrm{~s}, 1 \mathrm{H}, \mathrm{SO}_{2} \mathrm{NH}\right), 9.43$ $(\mathrm{s}, 1 \mathrm{H}, \mathrm{CONH}), 8.76(\mathrm{~d}, 1 \mathrm{H}$ thiazole ring), 8.23-7.24 (m, $12 \mathrm{H}, \mathrm{Ar}-\mathrm{H}), 6.62(\mathrm{~d}, 1 \mathrm{H}$ thiazole ring $), 2.86-1.83(\mathrm{~m}, 8 \mathrm{H}$, $\left.4 \mathrm{CH}_{2}\right), 2.38(\mathrm{~s}, 1 \mathrm{H}, \mathrm{CH}), 1.30\left(\mathrm{~s}, 6 \mathrm{H}, 2 \mathrm{CH}_{3}\right) .{ }^{13} \mathrm{C} \mathrm{NMR}$ $\left(\mathrm{DMSO}_{\mathrm{d}}\right): \delta / \mathrm{ppm} 173.54(\mathrm{Ar}-\mathrm{C}=\mathrm{O}), 169.15 \quad(\mathrm{~N}-\mathrm{C}=\mathrm{O})$, $147.34(\mathrm{C}=\mathrm{N}), 166.74-115.20$ (Ar-C and thiazole-C), 90.62 (spiro carbon), $52.91\left(2 \mathrm{CH}_{2}\right), 47.24(\mathrm{CH}), 31.85\left(2 \mathrm{CH}_{2}\right)$, $27.46\left(2 \mathrm{CH}_{3}\right)$. MS: $\left(\mathrm{M}^{+}=514\right)$; Anal. Calcd for $\mathrm{C}_{23} \mathrm{H}_{26} \mathrm{~N}_{6} \mathrm{O}_{4} \mathrm{~S}_{2}$ (514.63): C 53.68\%, H 5.09\%, N $16.33 \%$. Found: C 53.45\%, H 4.97\%, N 16.22\%.

4-Benzoylamino-8-bezyl-3-(2-furyl)-l-[4-(thiazol-2-ylsulfonyl)phenyl]-l,2,4,8-tetraazaspiro-[4.5]dec-2-ene 4p: Yield $62 \%$, m.p. $204-206^{\circ} \mathrm{C}$ (ethanol). IR, v/cm ${ }^{-1}: 3372$, 3340, 2356 (NH's), 1665, $1670\left(\mathrm{C}=\mathrm{O}^{\prime} \mathrm{s}\right), 1616(\mathrm{C}=\mathrm{N}), 1345$, $1147\left(\mathrm{SO}_{2}\right) ;{ }^{1} \mathrm{H} \mathrm{NMR}, \delta / \mathrm{ppm}: 11.61\left(\mathrm{~s}, 1 \mathrm{H}, \mathrm{SO}_{2} \mathrm{NH}\right), 9.45(\mathrm{~s}$, $1 \mathrm{H}, \mathrm{CONH}), 8.76(\mathrm{~d}, 1 \mathrm{H}$ thiazole ring), 8.26-7.23 (m, $12 \mathrm{H}$, Ar-H), 6.62 (d, $1 \mathrm{H}$ thiazole ring), 3.36 (s, $\left.2 \mathrm{H}, \mathrm{PhCH}_{2}\right), 2.83-$ $1.81\left(\mathrm{~m}, 8 \mathrm{H}, 4 \mathrm{CH}_{2}\right) ;{ }^{13} \mathrm{C}$ NMR $\left(\mathrm{DMSO}-\mathrm{d}_{6}\right): \delta / \mathrm{ppm} 173.65$ $(\mathrm{Ar}-\mathrm{C}=\mathrm{O}), 169.27(\mathrm{~N}-\mathrm{C}=\mathrm{O}), 147.34(\mathrm{C}=\mathrm{N}), 166.7-115.20$ (Ar-C and thiazole-C), 90.50 (spiro carbon), $52.89\left(2 \mathrm{CH}_{2}\right)$, $49.87\left(\mathrm{PhCH}_{2}\right), 32.20\left(2 \mathrm{CH}_{2}\right)$. MS: $\left(\mathrm{M}^{+} \cdot=562\right)$; Anal. Calcd for $\mathrm{C}_{27} \mathrm{H}_{26} \mathrm{~N}_{6} \mathrm{O}_{4} \mathrm{~S}_{2}$ (562.67): C 57.64\%, H 4.66\%, N 14.94\%. Found: C $57.85 \%$, H $4.53 \%$, N $15.05 \%$.

4-Benzoylamino-8-methyl-l-[4-(pyrimidin-2-ylsulfonyl)phenyl]-3-(2-thienyl)-l,2,4,8-tetraaza-spiro[4.5]dec-2-ene $4 q$ : $(1.33 \mathrm{~g}, 64 \%)$, m.p. $213-215^{\circ} \mathrm{C}$ (ethanol). IR, v/cm ${ }^{-1}: 3368,3346,2360$ (NH's), 1660, 1667 (C=O's), $1615(\mathrm{C}=\mathrm{N}), 1346,1153\left(\mathrm{SO}_{2}\right) ;{ }^{1} \mathrm{H} \mathrm{NMR}, \delta / \mathrm{ppm}: 11.60(\mathrm{~s}$, $\left.1 \mathrm{H}, \mathrm{SO}_{2} \mathrm{NH}\right), 9.44(\mathrm{~s}, 1 \mathrm{H}, \mathrm{CONH}), 8.86(\mathrm{~d}, 2 \mathrm{H}$ pyrimidine ring), 8.36-7.16 (m, 12H, Ar-H), 6.89 ( $\mathrm{t}, 1 \mathrm{H}$ pyrimidine ring), 2.86-1.83 (m, 8H, 4CH 2$), 2.31\left(\mathrm{~s}, 3 \mathrm{H}, \mathrm{NCH}_{3}\right) ;{ }^{13} \mathrm{C} \mathrm{NMR}$ $\left(\right.$ DMSO-d $\left._{6}\right): \delta=174.54(\mathrm{Ar}-\mathrm{C}=\mathrm{O}), 169.28(\mathrm{~N}-\mathrm{C}=\mathrm{O}), 147.63$ $(\mathrm{C}=\mathrm{N}$ ), 167.9-110.37 (Ar-C and pyrimidine-C), 89.95 (spiro carbon), $52.70\left(2 \mathrm{CH}_{2}\right), 46.64\left(\mathrm{CH}_{3}\right), 31.90\left(2 \mathrm{CH}_{2}\right)$. MS: $\left(\mathrm{M}^{+}\right.$. = 497); Anal. Calcd for $\mathrm{C}_{22} \mathrm{H}_{23} \mathrm{~N}_{7} \mathrm{O}_{3} \mathrm{~S}_{2}$ (497.60): $\mathrm{C} 53.10 \%, \mathrm{H}$
4.66\%, N 19.70\%. Found: C 52.87\%, H 4.78\%, N 19.57\%.

4-Benzoylamino-8-isopropyl-l-[4-(thiazol-2-yl-sulfonyl)phenyl]-3-(2-thienyl)-l,2,4,8-tetraazaspiro-[4.5]dec-2-ene 4r: Yield 66\%, m.p. 221-223 ${ }^{\circ} \mathrm{C}$ (ethanol).. IR, v/ $\mathrm{cm}^{-1}$ : 3374, 3347, 2362 (NH's), 1660, 1668 (C=O's), $1613(\mathrm{C}=\mathrm{N}), 1347$, $1151\left(\mathrm{SO}_{2}\right) ;{ }^{1} \mathrm{H} \mathrm{NMR}, \delta / \mathrm{ppm}: 11.61\left(\mathrm{~s}, 1 \mathrm{H}, \mathrm{SO}_{2} \mathrm{NH}\right), 9.47$ (s, $1 \mathrm{H}, \mathrm{CONH}), 8.76(\mathrm{~d}, 1 \mathrm{H}$ thiazole ring $), 8.38-7.16(\mathrm{~m}, 12 \mathrm{H}$, Ar-H), 6.62 (d, $1 \mathrm{H}$ thiazole ring), 2.82-1.83 (m, 8H, 4CH $)$ $2.33(\mathrm{~s}, 1 \mathrm{H}, \mathrm{CH}), 1.26\left(\mathrm{~s}, 6 \mathrm{H}, 2 \mathrm{CH}_{3}\right) ;{ }^{13} \mathrm{C} \mathrm{NMR}\left(\mathrm{DMSO}-\mathrm{d}_{6}\right)$ : $\delta / p p m 174.60(\mathrm{Ar}-\mathrm{C}=\mathrm{O}), 169.18(\mathrm{~N}-\mathrm{C}=\mathrm{O}), 147.34(\mathrm{C}=\mathrm{N})$, 166.76-115.20 (Ar-C and thiazole-C), 89.90 (spiro carbon), $52.80\left(2 \mathrm{CH}_{2}\right), 47.20(\mathrm{CH}), 32.20\left(2 \mathrm{CH}_{2}\right), 27.46\left(2 \mathrm{CH}_{3}\right) . \mathrm{MS}$ : $\left(\mathrm{M}^{+}=\right.$530); Anal. Calcd for $\mathrm{C}_{23} \mathrm{H}_{26} \mathrm{~N}_{6} \mathrm{O}_{3} \mathrm{~S}_{3}$ (530.69): $\mathrm{C}$ $52.06 \%$, H 4.94\%, N $15.84 \%$. Found: C 51.83\%, H 5.10\%, N $15.72 \%$.

4-Benzoylamino-8-benzyl-l-[4-(pyrimidin-2-yl-sulfonyl)phenyl]-3-(2-thienyl)-l,2,4,8-tetraazaspiro-[4.5]-dec-2-ene $4 s$ : Yield $65 \%$, m.p. $207-209^{\circ} \mathrm{C}$ (ethanol). IR, v/cm ${ }^{-1}: 3370$, 3341, 2356 (NH's), 1660, 1665 (C=O's), $16015(\mathrm{C}=\mathrm{N}), 1342$, $1147\left(\mathrm{SO}_{2}\right)$; ${ }^{1} \mathrm{H}$ NMR, $\delta / \mathrm{ppm}$ : $11.60\left(\mathrm{~s}, 1 \mathrm{H}, \mathrm{SO}_{2} \mathrm{NH}\right), 9.43$ (s, $1 \mathrm{H}, \mathrm{CONH}), 8.86(\mathrm{~d}, 2 \mathrm{H}$ pyrimidine ring), 8.37-7.18 (m, $12 \mathrm{H}, \mathrm{Ar}-\mathrm{H}), 6.89$ (t, $1 \mathrm{H}$ pyrimidine ring), $3.40(\mathrm{~s}, 2 \mathrm{H}$, $\left.\mathrm{PhCH}_{2}\right), 2.90-1.80\left(8 \mathrm{H}, \mathrm{m}, 4 \mathrm{CH}_{2}\right) ;{ }^{13} \mathrm{C}$ NMR (DMSO-d $\left.\mathrm{d}_{6}\right): \delta=$ $174.56(\mathrm{Ar}-\mathrm{C}=\mathrm{O}), 169.16(\mathrm{~N}-\mathrm{C}=\mathrm{O}), 147.36(\mathrm{C}=\mathrm{N}), 167.92-$ 110.37 (Ar-C and pyrimidine-C), 89.85 (spiro carbon), 53.00 $\left(2 \mathrm{CH}_{2}\right), 49.94\left(\mathrm{PhCH}_{2}\right), 31.87\left(2 \mathrm{CH}_{2}\right) . \mathrm{MS}:\left(\mathrm{M}^{+} \cdot=573\right)$; Anal. Calcd for $\mathrm{C}_{28} \mathrm{H}_{27} \mathrm{~N}_{7} \mathrm{O}_{3} \mathrm{~S}_{2}$ (573.70): C 58.62\%, H 4.74\%, N $17.09 \%$. Found: C $58.40 \%$, H $4.88 \%$, N 16.95\%.

4-Benzoylamino-8-methyl-3-(2-naphthyl)-l-[4-(thiazol-2-ylsulfonyl)phenyl]-l,2,4,8-tetraazaspiro-[4.5]dec-2-ene 4t: Yield $58 \%$, m.p. $257-259^{\circ} \mathrm{C}$ (ethanol). IR, v/cm ${ }^{-1}: 3368,3342,2358$ (NH's), 1650, $1665(\mathrm{C}=\mathrm{O} ' \mathrm{~s}), 1597(\mathrm{C}=\mathrm{N}), 1350,1156\left(\mathrm{SO}_{2}\right)$; ${ }^{1} \mathrm{H}$ NMR, $\delta / p p m: 11.61$ (s, $\left.1 \mathrm{H}, \mathrm{SO}_{2} \mathrm{NH}\right), 9.41$ (s, $\left.1 \mathrm{H}, \mathrm{CONH}\right)$, $8.76(\mathrm{~d}, 1 \mathrm{H}$ thiazole ring), 8.83-7.25 (m, 16H, Ar-H), $6.62(\mathrm{~d}$, $1 \mathrm{H}$ thiazole ring), $3.00-1.81\left(\mathrm{~m}, 8 \mathrm{H}, 4 \mathrm{CH}_{2}\right), 2.36(\mathrm{~s}, 3 \mathrm{H}$, $\left.\mathrm{NCH}_{3}\right) ;{ }^{13} \mathrm{C}$ NMR (DMSO-d $\mathrm{d}_{6}$ ): $\delta /$ ppm $182.60 \quad(\mathrm{Ar}-\mathrm{C}=\mathrm{O})$, $168.86(\mathrm{~N}-\mathrm{C}=\mathrm{O}), 147.34(\mathrm{C}=\mathrm{N}), 166.7-115.20(\mathrm{Ar}-\mathrm{C}$ and thiazole-C), 90.12 (spiro carbon), 89.66 (spiro carbon), 52.87 $\left(2 \mathrm{CH}_{2}\right), 46.60\left(\mathrm{NCH}_{3}\right), 31.80\left(2 \mathrm{CH}_{2}\right)$. MS: $\left(\mathrm{M}^{+}=546\right)$; Anal. Calcd for $\mathrm{C}_{27} \mathrm{H}_{26} \mathrm{~N}_{6} \mathrm{O}_{3} \mathrm{~S}_{2}$ (546.67): C 59.32\%, H 4.79\%, N $15.37 \%$. Found: C $59.10 \%$, H $4.92 \%$, N $15.50 \%$.

4-Benzoylamino-8-methyl-3-(2-naphthyl)-l-[4-(pyrimidin2-yl-sulfonyl)phenyl]-l,2,4,8-tetraaza-spiro[4.5]-dec-2-ene $4 u$ : Yield $56 \%$, m.p. $263-265^{\circ} \mathrm{C}$ (ethanol). IR, v/cm ${ }^{-1}: 3372$, 3343, 2358 (NH's), 1662, 1652 (C=O's), $1614(\mathrm{C}=\mathrm{N}), 1351$, $1158\left(\mathrm{SO}_{2}\right)$; ${ }^{1} \mathrm{H} \mathrm{NMR}, \delta / \mathrm{ppm}: 12.60\left(\mathrm{~s}, 1 \mathrm{H}, \mathrm{SO}_{2} \mathrm{NH}\right), 9.40$ (s, $1 \mathrm{H}, \mathrm{CONH}), 8.82(\mathrm{~d}, 2 \mathrm{H}$ pyrimidine ring), 8.87-7.28 (m, $16 \mathrm{H}, \mathrm{Ar}-\mathrm{H}), 6.89$ (t, $1 \mathrm{H}$ pyrimidine ring), 3.01-1.80 (m, $8 \mathrm{H}$, $\left.4 \mathrm{CH}_{2}\right), 2.38\left(\mathrm{~s}, 2 \mathrm{H}, \mathrm{NCH}_{3}\right)$ ppm. ${ }^{13} \mathrm{C} \mathrm{NMR}\left(\mathrm{DMSO}-\mathrm{d}_{6}\right)$ : $\delta /$ ppm $182.62(\mathrm{Ar}-\mathrm{C}=\mathrm{O}), 168.82(\mathrm{~N}-\mathrm{C}=\mathrm{O}), 147.60(\mathrm{C}=\mathrm{N})$, 167.91-110.33 (Ar-C and pyrimidine-C), 89.68 (spiro carbon), $53.00\left(2 \mathrm{CH}_{2}\right), 46.64\left(\mathrm{CH}_{3}\right), 32.10\left(2 \mathrm{CH}_{2}\right)$. MS: $\left(\mathrm{M}^{+}\right.$. = 541); Anal. Calcd for $\mathrm{C}_{28} \mathrm{H}_{27} \mathrm{~N}_{7} \mathrm{O}_{3} \mathrm{~S}$ (541.64): C 62.09\%, $\mathrm{H}$ $5.02 \%$, N $18.10 \%$. Found: C 61.85\%, H 5.18\%, N 18.06\%.

\subsection{Screening for Antimicrobial Activity}

Antimicrobial activity screening of the synthesized 
compounds was determined by the agar dilution technique as recommended by the Clinical and Laboratory Standard Institute (CLSI) [30]. The tested compounds were dissolved in dimethyl sulfoxide (DMSO). An inoculum of about $1.5 \mathrm{x}$ $10^{8}$ colony forming unit per spot was applied to the surfaces of Mueller-Hinton agar plates containing graded concentrations of the respective compound; plates were incubated at $37{ }^{\circ} \mathrm{C}$ for $18 \mathrm{~h}$. The spot with the lowest concentration of compound showing no growth was defined as the minimum inhibitory concentration (MIC). All organisms used in this study were standard strains were obtained from the Microbiology laboratory (Al-Aqsa University) and included bacterial strain such as Enterococci, Escherichia coli, Staphylococcus aureus, Klebsiella spp, Proteus spp, and fungi strain such as Aspergillus niger, Candida albicans. The MIC of Tetracycline and fluconazole was determined concurrently as reference for antibacterial and antifungal activities, respectively (Table 1). Control DMSO was carried out with each experiment.

\section{Results and Discussion}

The nitrilimines 2 employed in this study was generated in situ from the corresponding hydrazonoyl halides 1 by the action of a suitable base as shown in Figure 1. The treatment of the resulting non-isolable nitrilimines 2, 1,3-dipole, with appropriate 1-methyl, 1-isopropyl and 1-benzyl-4-piperidone benzoyl-hydrazones 3 in 1,4-dioxane or tetrahydrofuran in presence of triethylamine as a base, gave in each case a single product that proved to be the respective $1,3,4$ trisubstituted 1,2,4-spirotriazole derivatives 4a-u, as cycloaddition products through the 1,3-dipolar cycloaddition of nitrilimines 2 to the exocyclic double bond $(\mathrm{N}=\mathrm{C})$ of benzoylhydrazone 3 instead of the spirotetrazine cyclocondensation products 5a-u (Figure 1). The purity of obtained compounds was controlled by TLC and elemental analysis. Both the analytical and spectral data (IR, ${ }^{1} \mathrm{H}$ NMR, ${ }^{13} \mathrm{C}$ NMR and mass spectra) of the synthesized spirotriazoles were in full agreement with the proposed structures.<smiles>CCCCCCCC(C)C</smiles>

1

i: $\mathrm{Et}_{3} \mathrm{~N} / 0^{\circ} \mathrm{C}$; ii: Dioxane / r.t.<smiles>[14C]c1nccs1</smiles>
$\mathrm{Ar} / \mathrm{Ar}^{\prime} / \mathrm{R}$ a, $\mathrm{Me} / 2$-thiazoly1/Me b, $\mathrm{Me} / 2$-thiazolyl/ $\mathrm{CHMe}_{2}$ c, $\mathrm{Me} / 2$-thiazolyl $/ \mathrm{CH}_{2} \mathrm{Ph}$

d, $\mathrm{Me} / 2$-pyrimidinyl/Me e, $\mathrm{Me} / 2$-pyrimidinyl- $-\mathrm{CHMe}_{2}$ f, $\mathrm{Me} / 2$-pyrimidinyl $/ \mathrm{CH}_{2} \mathrm{Ph}$ g, $\mathrm{Ph} / 2$-thiazolyl/Me

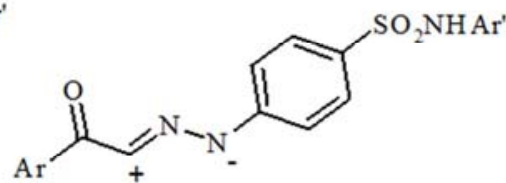<smiles>[Y]NN=C1CCN([R])CC1</smiles>

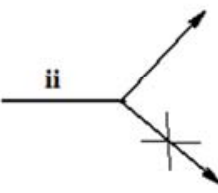

$\mathrm{Y}=\mathrm{PhCO}$

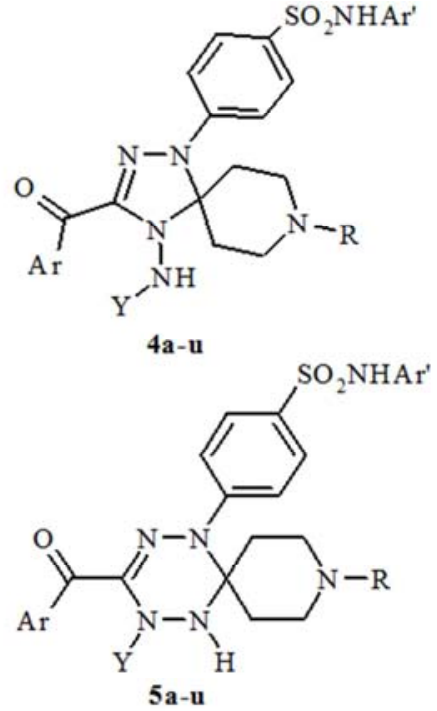

$\mathrm{Ar} / \mathrm{Ar} / \mathrm{R}$

h, $\mathrm{Ph} / 2$-thiazolyl/ $\mathrm{CHMe} 2$

i. $\mathrm{Ph} / 2$-thiazolyl $/ \mathrm{CHPh}$

j, $\mathrm{PhNH} / 2$-thiazolyl/Me

k, $\mathrm{PhNH} / 2$-thiazolyl $/ \mathrm{CH}_{2} \mathrm{Ph}$

l, $\mathrm{PhNH} / 2$-pyrimidinyl/CHMe2

m, $\mathrm{PhNH} / 2$-pyrimidinyl $/ \mathrm{CH}_{2} \mathrm{Ph}$

n, 2-Furyl/2-thiazolyl/Me
$\mathrm{Ar} / \mathrm{Ar} / \mathrm{R}$

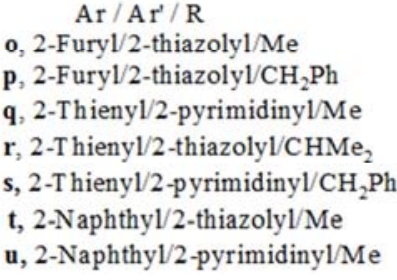

o, 2-Furyl/2-thiazolyl/Me

p, 2-Furyl/2-thiazolyl $/ \mathrm{CH}_{2} \mathrm{Ph}$

q, 2-Thienyl/2-pyrimidinyl/Me

r, 2-Thienyl/2-thiazolyl/CHMe,

t, 2-Naphthyl/2-thiazolyl/Me

u, 2-Naphthyl/2-pyrimidinyl/Me

Figure 1. Synthetic pathway of 1,2,4,8-tetaazspiro[4.5]dec-2-enes 4a-u.

\subsection{Spectroscopical Data of Spiro Compounds $4 a-u$}

The characterization data of synthesized spiro compounds $4 \mathrm{a}-\mathrm{u}$ are given in the experimental section. These compounds gave satisfactory analysis for the proposed structures which are confirmed on the bases of their spectroscopical data. The IR spectra showed the strong absorption band of CONH of the triazole ring in the region $3380-3360 \mathrm{~cm}^{-1}$, in addition to, characteristic band of $\mathrm{SO}_{2} \mathrm{NH}$ in the region of $3340-3220 \mathrm{~cm}^{-}$ 1 , $(\mathrm{Ar}-\mathrm{C}=\mathrm{O})$ at about $1680-1660 \mathrm{~cm}^{-1}$ and bands at 1350 , $1150 \mathrm{~cm}^{-1}$ attributed to $\mathrm{SO}_{2}$ of sulfonamide group. In the ${ }^{1} \mathrm{H}$
NMR spectra, a characteristic signal due to the PhCONH proton of the triazole ring appeared at 9.50-9.40 ppm. The $\mathrm{NH}$ of the tetrazine structures $5 \mathrm{a}-\mathrm{u}$ if formed is expected to resonate at about 5-4 ppm [43]. The signals at 8.70, 6.60 ppm and $8.40,6.80 \mathrm{ppm}$ are attributed to thiazole and pyrimidine protons, respectively. Also, the spectra exhibit a characteristic singlet at $11.6-11.5 \mathrm{ppm}$ and due to $\mathrm{SO}_{2} \mathrm{NH}$ proton. The structures of compounds $4 \mathrm{a}-\mathrm{u}$ were further confirmed by ${ }^{13} \mathrm{C}$ NMR spectra, which account for the different carbons of these spirotriazoles. The signal at 89-91 ppm was attributed to the C-5 (spiro carbon) of the triazole 
ring is of special significance. This assignment is in good agreement with literature data for quaternary carbons flanked by two nitrogen atoms in five-membered heterocycles $[36,37,41]$, which provides strong evidence in support of the structures $4 \mathrm{a}-\mathrm{u}$ rather than the six-membered structures 5a-u, which is expected to have a spiro carbon at about 60-70 ppm. The signal at about $147-146 \mathrm{ppm}$ was attributed to the C-3 carbon of the triazole ring, and this is similar to reported values of azomethine carbons of five-membered hete rocycles [37]. The electron impact (EI) mass spectra displayed the correct molecular ions $\left(\mathrm{M}^{+}\right)$in accordance with the suggested structures. The base peak in all these compounds $4 \mathrm{a}-\mathrm{u}$ was that of the conjugated vinyl triazole cation this fragmentation pattern is well known for cycloalkanes [44].

\subsection{Antimicrobial Activity}

Most of the synthesized compounds were screened in vitro for their antimicrobial activity against a variety of bacterial strains such as Enterococci, Escherichia coli, Staphylococcus aureus, Klebsiella spp, Proteus spp, and fungi such as Aspergillus niger, Candida albicans, employing the nutrient agar disc diffusion method [30] at $1-100 \mathrm{mg} / \mathrm{ml}$ concentration in dimethyl sulfoxide (DMSO) which used as solvent control, by measuring the average diameter of the inhibition zone in $\mathrm{mm}$. The results are given in Table 1 .

All the experiments were carried out in triplicate. The results showed that all the tested compounds exhibited good degree of activity against different strains of bacteria and fungi compared with well-known antibacterial and antifungal substances such as tetracycline and fluconazole respectively. According to National Committee for Clinical Laboratory Standards (NCCLS, 2004) methodology, zones of inhibition for tetracycline and fluconazole $<14 \mathrm{~mm}$ were considered resistant, between 15 and $18 \mathrm{~mm}$ were considered weakly sensitive and $>19 \mathrm{~mm}$ were considered sensitive. The presence of sulfonamide moieties showed a better spectrum of activity than the reference drug.

Table 1. Antimicrobial screening results of the tested compounds*.

\begin{tabular}{|c|c|c|c|c|c|c|c|}
\hline \multirow{2}{*}{$\begin{array}{l}\text { Comp. } \\
\text { No. }\end{array}$} & \multicolumn{4}{|c|}{ Antibacterial activity } & \multicolumn{3}{|c|}{ Antifungal activity } \\
\hline & Enter. & E. coli & S. aureus & K. spp & P. spp & C. alb. & A. niger \\
\hline $4 a$ & 16 & 18 & 16 & 16 & 15 & 19 & 18 \\
\hline $4 b$ & 18 & 19 & 14 & 15 & 17 & 20 & 19 \\
\hline $4 \mathrm{c}$ & 17 & 14 & 15 & 17 & 14 & 18 & 18 \\
\hline $4 d$ & 16 & 19 & 17 & 18 & 15 & 20 & 19 \\
\hline $4 \mathrm{e}$ & 17 & 17 & 17 & 19 & 13 & 19 & 19 \\
\hline $4 \mathrm{f}$ & 15 & 14 & 15 & 18 & 16 & 17 & 18 \\
\hline $4 g$ & 19 & 16 & 14 & 15 & 19 & 16 & 15 \\
\hline $4 \mathrm{~h}$ & 15 & 18 & 13 & 16 & 15 & 14 & 17 \\
\hline $4 \mathrm{i}$ & 16 & 16 & 17 & 14 & 14 & 18 & 16 \\
\hline $4 j$ & 14 & 14 & 16 & 15 & 16 & 16 & 17 \\
\hline $4 \mathrm{k}$ & 16 & 17 & 14 & 13 & 15 & 17 & 15 \\
\hline 41 & 17 & 15 & 13 & 15 & 17 & 18 & 14 \\
\hline $4 m$ & 19 & 17 & 18 & 19 & 18 & 19 & 18 \\
\hline $4 n$ & 17 & 15 & 15 & 16 & 15 & 16 & 16 \\
\hline 40 & 19 & 19 & 14 & 16 & 19 & 17 & 17 \\
\hline $4 q$ & 17 & 20 & 18 & 17 & 18 & 14 & 18 \\
\hline $4 r$ & 16 & 18 & 16 & 15 & 18 & 15 & 19 \\
\hline $4 s$ & 15 & 17 & 18 & 17 & 16 & 14 & 16 \\
\hline $4 u$ & 19 & 18 & 19 & 16 & 19 & 16 & 18 \\
\hline DMSO & -- & -- & -- & -- & -- & -- & -- \\
\hline
\end{tabular}

*Calculated as average of three values.

Enter. $=$ Enterococci, E. coli $=$ Escherichia coli, S. aureus $=$ Staphylococcus aureus, K. spp =Klebsiella spp, P. spp $=$ Proteus spp, C. alb. $=$ Candida albicans, A. niger $=$ Aspergillus niger.

\section{Conclusion}

The reaction of nitrilimines 2 with 4-piperidone benzoylhydrazones 3 leads to formation of novel heterocyclic spirotriazoles $4 \mathrm{a}-\mathrm{u}$ containing 1,2,4-triazole, piperidine and sulfonamide moieties and evaluated for antibacterial, and antifungal activities. From the screening results, it found to possess various antimicrobial activities towards all the microorganisms tested. The results confirm that, the antimicrobial activity is strongly dependent on the nature of the substituents on triazole nucleus. The pyrimidinyl, thiazolyl derivatives generally led to improvements in activity against both bacteria and fungi. The present study can lead medicinal chemists to design and synthesize similar compounds with enhanced biological potency in future.

\section{Acknowledgements}

The authors are great thankful to the Qatar Charity for the financial support of this research through Ibhath grant.

\section{References}

[1] Sayed, A. R. Tetrahedron 2012, 68(13), 2784-2789.

[2] Sayed, A. R.; Wiggins, J. S. J. Appl. Poly. Sci. 2011, 120(2), 623-630.

[3] Sayed, A. R. Tetrahedron Lett. 2010, 51, 4490-4493. 
[4] Sayed, A. R.; Wiggins, J. S. Polymer 2008, 49(9), 2253-2259.

[5] G. Periyasami, R. Raghunathan, G. Surendiran, N. Mathivanan, Eur. J. Med. Chem., 2009, 44, 959.

[6] K. Amin, M. Kamel, M. Anwar, M. Khedr, Y. M. Syamb, Eur. J. Med. Chem., 2010, 45, 2117.

[7] R. R. Kumar, S. Perumal, S. C. Manju, P. Bhatt, P. Yogeeswari and D. Sriram, Bioorg. Med. Chem. Lett., 2009, 19, 3461.

[8] A. Dandia, R. Singh, S. Khaturia, C. Merienne, G. Morgant and A. Loupy, Bioorg. Med. Chem., 2006, 14, 2409.

[9] G. Lang, A. Pinkert, J. W. Blunt and M. H. G. Munro, J. Nat. Prod., 2005, 68,1796.

[10] C. A. Maier and B. Wuensch, J. Med. Chem., 2002, 45, 438.

[11] M. Wolf, A. A. Masciffi, US Patent, 1968, 395, 156; Chem. Abstr., 1988, 69, 96504r.

[12] H. C. Rohm, British Patent, 1962, 913, 937; Chem. Abstr. $1963,59,577 \mathrm{f}$.

[13] G. Winters and N. D. Mola, German Patent, 1975, 2442, 667; Chem. Abstr. 1975, 83, 28096.

[14] M. J. Kornet and A. P. Thio, J. Med. Chem., 1976, 19, 892.

[15] S. Gurvinder, Maninderjit K., Mohan C., Int Res J Pharm. 2013, 4, 82

[16] H. C. Patil, Patil I. M., Brose S. L, Pawar S. P, World J Pharm Pharmaceut Sci. 2015, 4:532

[17] P. B. Mohite, Bhaskar V. H, Int J PharmTech Res., 2011, 3, 1557

[18] N. A. Kheder, S. M. Riyadh, A. M. Asiry, Chem. Pharm. Bull. 2013, 61 (5) 504-510.

[19] Y. Naito, F. Akahoshi, S. Takeda, T. Okada, M. Kajii, H. Nishimura, M. Sugiura, C. Fukaya, Y. Kagitani, J. Med. Chem., 1996, 39, 3019.

[20] K. Okonogi and A. Tasaka, Can. Pat. Appl. CA 2132791 (Chem. Abstr., 1996, 124, 8821t).

[21] S. M. Kudari, S. M. Beede, W. Munera, Asian J. Chem., 1997, 9, 20.

[22] T. Denzel and H. Hoehn, U. S. Patent 1976, 3971801 (Chem. Abstr., 1977, 86, P16675k).

[23] A. H. El-Masry, H. H. Fahmy, S. H. Ali and A. S. Waheed, Molecules, 2000, 5, 1429.

[24] A. S. Orabi, M. A. Moneim, E. E. Salem, M. E. Abdel-Fattah, Polish J. Chem., 2000, 74, 1675.
[25] A. Almasirad, S. A. Tabatabai, M. Faizi, A. Kebriaeezadeh, N. Mehrabi, A. Dalvandi and A. Shafiee, Bioorg. Med. Chem Lett., 2004, 14, 6057.

[26] T. George, D. Mehta, R. Tahilramani, J. David, P. Talwalker, $J$. Med. Chem., 1971, 14, 335.

[27] J. Bailey, J. Chem. Soc. Perkin Trans. 1, 1977, 2047.

[28] T. Farghaly, M. Abdallah, G. Masaret, Z. Muhammad, Eur. J. Med. Chem., 2015, 97, 320.

[29] N. Abdel Hafez, T. Farghaly, M. Al-Omar, M. Abdalla, Eur. J. Med. Chem., 2010, 45, 4838.

[30] T. A. Farghaly and H. K. Mahmoud, Arch. Pharm. Chem. Life Sci., 2013, 346, 392.

[31] Girgis, A. S., Barsoum, F. F., Samir, A. Eur. J. Med. Chem. 2009, 44, 2447-2451.

[32] Dalloul, H. M. Arkivoc, 2008 (xiv) 234-241.

[33] Dalloul, H. M.; Abu samaha, A. J Serb Chem. Soc., 2010, 75, 1473-1479.

[34] Dalloul, H. M.; Boyle, P. H. Heterocycl. Commun. 2003, 9, 507-514.

[35] Dalloul, H. M. Chem. Heterocycl. Comp. 2004, 40, 14021407.

[36] Dalloul, H. M.; Boyle, P. H. Turk. J. Chem. 2006, 30, 119-124.

[37] Dalloul, H. M.; Boyle, P. H. Heterocycl. Commun. 2007, 13, 155-160.

[38] Thaher B. A.; Otto, H-H., Monatsh. Chem. 2002, 133, 10111016.

[39] H. Hassaneen, Shawali, A., Elwan N., Abounada, N., Sulfur Lett. 1992, 13, 273-285.

[40] H. Hassaneen, A. Shawali, N. Elwan, N. Abounada, Org. Prep. Proced. Int. 1992, 24,171-175.

[41] H. Dalloul, K. El-nwairy, A. Shorafa A. Abu Samaha, South. Braz. J. Chem. 2016, Accepted.

[42] M. Okimoto, T. Chiba, J. Org. Chem., 1990, 55, 1070.

[43] H. M. Dalloul, South. Braz. J. Chem. 2015, 22, 17-26.

[44] M. Hesse, H. Meier, B. Zech, Spectroscopic Methods in Organic Chemistry, George Thieme Verlag, Stuttgart, 1997, p. 229. 